Helgoländer wiss. Meeresunters. 19, 205-215 (1969)

\title{
Das asymptotische Wachstum der Fische - ein Nonsens?
}

\author{
F. KRÜGER \\ Biologische Anstalt Helgoland, Zentrale, Hamburg 50
}

\begin{abstract}
The asymptotic growth of fishes - a nonsense? Considering an incomplete growth curve of the cod Gadus morbua, KNIGHT (1968) questioned the asymptotic curvature of growth in fishes, which easily may be shown in complete growth curves illustrating the attainment of maximum size. It is also possible to calculate maximum size employing the FORD-WALFORD formula. Maximum size, calculated in this manner, may also be used in the BERTALANFFY function. For other mathematical growth formulations different maximum sizes would be obtained. The numerical value of the maximum size depends upon the mathematical interpretation of the growth process. Therefore it always represents a mathematical parameter without biological meaning. It is shown that data of KETCHEN (ForREsTER 1966) on growth in $\hat{\delta} \hat{O}$ of the flat fish Eopsetta jordani may be much better represented by the new growth formula (KRÜGER 1965) than by the BERTALANFFY function.
\end{abstract}

\section{EINLEITUNG}

Vor kurzem veröffentlichte KNIGHT (1968) eine Arbeit, in der er glaubt, die asymptotische Annäherung des Wachstums der Fische an einen Endwert als eine unhaltbare Annahme hinstellen zu können. Die Frage ist dadurch bedeutungsvoll, daß bei den Versuchen zu einer mathematischen Darstellung des Fischwachstums eine Endgröße in mehreren Formulierungen als Parameter auftritt.

Dieser Einwand eines Mathematikers ist ernster zu nehmen, da nur wenige Biologen mit der Wachstumsmathematik so weit vertraut sind, daß sie die Unzulässigkeit dieser Behauptung aus eigener Erfahrung erkennen können. Gegner einer mathematischen Behandlung biologischer Probleme können in ihrer ablehnenden Haltung durch das Urteil eines scheinbaren Fachmannes bekräftigt werden.

KNIGHT zieht zum Vergleich und zur Bestätigung seiner Ansicht eine menschliche Wachstumskurve heran, bei der er bereit ist, die Annäherung an einen Grenzwert anzuerkennen. Er versäumt allerdings, den asymptotischen Charakter dieser Annäherung exakt zu belegen. In der Tat läßt das Längenwadhstum der Säugetiere wenigstens größenordnungsmäßig die Annäherung an eine Endgröße erkennen. Es ist dieses eine Erscheinung, die allgemein bekannt ist. Mit dem Einsetzen der Pubertät wird die Wachstumsgeschwindigkeit herabgesetzt, bis sie schließlich wahrscheinlich unmeßbar gering wird. Das Wachstum des Menschen und der Säugetiere und anderer Formen mit begrenztem Wachstum läßt sich aber bislang mit keiner der vorgeschlagenen Formulie- 
rungen mit einheitlichen Parametern von der Geburt bis zum Abschluß des Wachstums befriedigend darstellen. Die Schwierigkeit hierbei bereitet die Unstetigkeit zur Zeit der Pubertät. Wollen wir mit einfacheren Formulierungen arbeiten, die keinen allzu bohen Aufwand an Parametern benötigen, bleibt kein anderer Ausweg übrig, als eine Unstetigkeit anzunehmen und das postnatale Wachstum der Säugetiere in zwei Phasen zu unterteilen: das puerale (kindliche) und das postpuerale Wachstum nach der Pubertät. Vom rein mathematischen Standpunkt gesehen, erscheint das vielleicht nicht als eine ideale Lösung. Der Aufwand an einzusetzenden Parametern ist aber immer noch geringer, als er bei einem geeigneten Polynom wäre. Die Unstetigkeit in den Wachstumskurven der Säugetiere bei der Pubertät ist schon länger bekannt (vgl. z. B. voN BertalanfFy 1938). Ich habe sie selbst später bestätigt und gezeigt, daß man mit ihrer Annahme auch das menschliche Wachstum befriedigend darstellen kann (KRüGER 1966). Durch die Umstellung des Hormonhaushaltes zu dieser Zeit erscheint diese Unstetigkeit auch physiologisch verständlich. Wir mïssen uns bei der mathematischen Formulierung biologischer Phänomene - zunächst wenigstens - damit begnügen, beschränkte Bereiche zu erfassen. Nach beiden Seiten hin extrapolierbare Formulierungen besitzen wir noch nicht. Sie sind wahrscheinlich auch eine Utopie. Auch die durch ihre Exaktheit beispielliaften physikalischen Formulierungen haben oft nur einen begrenzten Anwendungsbereich.

\section{DAS UNBEGRENZTE WACHSTUM DER FISCHE}

Der andersartige Verlauf der Wachstumskurven der Säugetiere verbietet es grundsätzlich, sie zum Vergleich mit Fischen heranzuziehen. Das Wachstum der Fische verläuft nach dem Abschluß der larvalen Entwicklung ohne Unstetigkeit. Sie besitzen wie seit langem bekannt - ein unbegrenztes Wachstum, das heißt, sie wachsen solange sie leben. Das bedeutet aber nicht, daß ein Goldfisch zu einer beliebigen Größe heranwachsen kann. Das Wachstum jedes Fisches bleibt innerhalb artgebundener Dimensionen.

KNIGHT (1968) stützt seine These von dem Fehlen einer Endgröße bei Fischen auf eine Zahlenreihe von Graham (1933) für das Wachstum des Kabeljau (Gadus morbua), das er mit einem gleichmäßigen jährlichen Zuwachs von etwa $14 \mathrm{~cm}$ befriedigend wiedergeben $\mathrm{zu}$ können glaubt. Rechnet man nun aber mit einem solchen linearen $\mathrm{Zu}$ wachs die theoretischen Längen für die einzelnen Altersstufen aus, so kommt man zu Abweichungen bis zu $16 \%$ von den beobachteten Werten, eine Abweichung, die bei einer so kurzen Zahlenreihe vollkommen unzulässig ist, selbst wenn man die Unschärfe der Meßdaten berücksichtigt. Die Erfahrung hat gezeigt, daß einfache lineare Beschreibungen des Fischwachstums für eine allgemeinere Anwendung ungeeignet sind. KNIGHT kommt aber auf Grund seiner vollkommen unzulässigen Vereinfachung zu der Anschauung:,.. it is not at all evident from the data alone at what length the growth stops or even that it stops at all ..."

Wenn man zu befriedigenden Ergebnissen kommen will, darf man auf keinen Fall die in der Wachstumskurve vorhandene und auch von dem Autor erwähnte Krümmung 
vernachlässigen und an ihrer Stelle eine Gerade annehmen. Gerade in der Erfassung dieser Krümmung liegt die Schwierigkeit, welche die mathematische Darstellung von Wachstumskurven bereitet. Dem Autor wäre die Fehlerhaftigkeit seines Vorgehens vielleicht eher bewußt geworden, wenn er seiner Kritik nicht die sehr unvollständige Zahlenreihe von GRAHAm zugrunde gelegt hätte, sondern eine Zahlenreihe benutzt hätte, die eine wesentlich größere Altersspanne umfaßt. Solche liegen in der Literatur vor. Abbildung 1 zeigt die graphische Darstellung des Wachstums des Kabeljau nach den Daten von Hansen (1949) über eine Spanne von 14 Jahren. Diese Darstellung

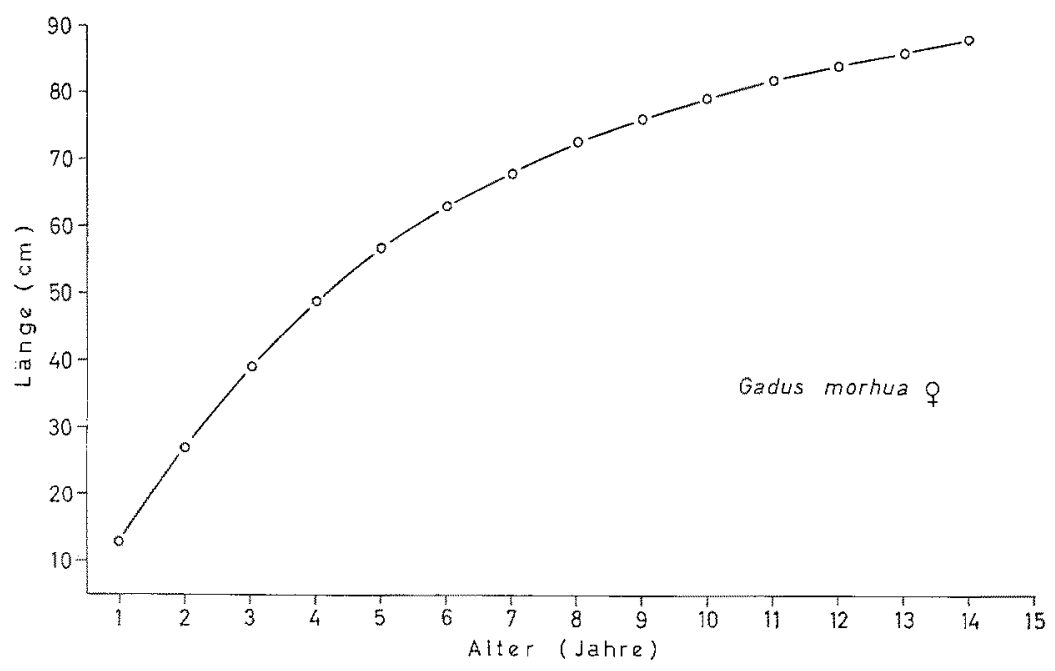

Abb. 1: Lineare Darstellung des Wachstums des Kabeljau (Gadus morbua, 9 ) nach Daten von HaNSEN (1949) für den Julianehaab- und Frederikshaab Distrikt

zeigt eindeutig die für Wachstumskurven charakteristische Krümmung, und niemand könnte es mit gutem Gewissen verantworten, sie durch eine Ausgleichsgerade zu ersetzen. Ubrigens würde die von KNIGHT angenommene Wachstumsrate von $14 \mathrm{~cm}$ je Jahr für die ältesten Fische der Kurve eine Länge von etwa $2 \mathrm{~m}$ ergeben. Kabeljau dieser Länge sind noch nie beobachtet worden.

Außerdem liefert die lineare Wachstumsdarstellung, auf der KNIGHT seine Kritik aufbaut, dadurch kein zutreffendes mathematisches Bild, da man die Zuwachsrate eines Organismus auf die jeweils gegebene Größe beziehen muß. Bei einem Fisch von $18 \mathrm{~cm}$ Länge bildet ein Zuwachs von $14 \mathrm{~cm}$ eine Streckung um $78 \%$, bei einem Fisch von $90 \mathrm{~cm}$ Länge bedeutet der gleiche Zuwachs eine Streckung um nur $16 \%$. Hier wird die Abnahme der Wachstumsrate mit zunehmendem Alter deutlich. Sie muß in einer befriedigenden Wachstumsbeschreibung enthalten sein; sie fehlt in einer linearen Darstellung.

Graphisch stellt man die auf die jeweilige Ausgangsgröße bezogene Wachstumsrate sehr einfach dar, wenn man die Logarithmen der Dimension als Funktion des linearen Alterswertes wiedergibt. 


\section{DER MAXIMALWERT IN WACHSTUMSFUNKTIONEN}

Die Behauptung des Autors, das Fischwachstum zeige keine asymptotische Annäherung an einen Grenzwert, läßt sich also schon durch einfachste graphische Darstellungen widerlegen. Es bleibt die Frage zu klären, ob die Einsetzung eines solchen Endwertes in mathematische Formulierungen gerechtfertigt ist. KNIGHT beruft sich hierbei auf eine kürzlich erschienene Auswertung von KETCHEN \& FORRESTER (1966) für das Wachstum des Plattfisches Eopsetta jordani. Für diesen Fisch berechnen die Autoren die Parameter der BERTALANFFY-Funktion und erzielen vor allem bei den 9 of einen sehr befriedigenden Anschluß an ihre Messungen.

Die Autoren geben in ihrer Arbeit auch die WALForD-Darstellung ihrer Daten, die für die $q Q$ in sehr guter Näherung eine Gerade liefert, bei den $\delta 3$ allerdings nur oberhalb der Altersstufe 4.

Wenn KNIGHT dayon spricht, bei der WALFORD-Darstellung würden die Werte auf eine Gerade gezwungen, so kann man diese Behauptung als Nonsens bezeichnen und sie ist unverständlich, da kurz vorher die sicher nicht auf einer Geraden liegenden Punkte für das Wachstum des Kabeljau vom Autor auf eine Gerade gezwungen wurden. Der WALFORD-Darstellung liegt die Funktion zugrunde:

$$
1_{(n+1)}=a+b \cdot 1_{n}
$$

Die WALForD-Beziehung besagt, daß bei gleichmäßig gewählten Zeitabständen die Zuwachsraten in zwei aufeinanderfolgenden Perioden in einem konstanten Verhältnis zueinander stehen. Die Richtigkeit dieses Prinzips läßt sich durch eine sehr große Zahl von Beispielen belegen und gestatter, gegebene Wachstumsreihen mit sehr großer Genauigkeit numerisch wiederzugeben. Falls KNIGHT Zweifel an der Berechtigung der Geradlinigkeit gehabt hätte, wäre es richtiger gewesen, wenn er mit Hilfe der Korrelationsrechnung an gegebenen Beispielen gezeigt hätte, daß die Darstellung der Meßwerte durch eine Regressionsgerade unzulässig ist.

Die WALFORD-Darstellung schließt offensichtlich mathematische Prinzipien ein, die vielen Wachstumsvorgängen zugrunde liegen. Gelingt es nun, quantitative Zusammenhänge, deren mathematischer Zusammenhang ungeklärt ist, durch geeignete Variationen der Koordinatenteilungen in eine Gerade zu überführen, so ist mit der Gleichung dieser Geraden ein Schlïssel zur Aufstellung der zugrundeliegenden Funktion gegeben. In der Physik ist das Prinzip, durch Variation der Koordinatenteilungen Kurven unbekannter Funktion in eine Gerade zu überführen, ein bekanntes und mit Erfolg angewandtes Verfahren. Warum sollte es sich nicht auch in der Biologie als nützlich erweisen? Wenn KNIGHT bei seiner Modifikation der WaLFord-Darstellung zu einer Kurve kommt, so kann man diese Modifikation nur als einen Rückschritt bezeichnen, es sei denn, er zeigt, daß die seiner Kurve zugrundeliegende mathematische Funktion eine bessere Annäherung an gegebene Meßreihen gestattet, als die WALFord-Formel.

FORD (1933), der schon lange vor WALFORD (1946) diese Gesetzmäßigkeit in der Folge von Daten für das Längenwachstum aufdeckte, erkannte auch, daß seiner Art der Darstellung die geometrische Reihe zugrunde liegt:

$$
1_{n}=a\left(1+b+b^{2}+b^{3}+\ldots b^{n-1}\right)+1_{0} \cdot b^{n}
$$


$(1=$ Länge, die Indizes $\mathrm{o}-\mathrm{n}$ geben das Alter an, $\mathrm{b}$ ist die Steigung der Regressionsgeraden; $a$ ist das additive Glied der FoRD-WALFORD-Formel.)

$\mathrm{Da}$ der Wert von b stets unter 1 liegt, hat die Summe der Exponentialglieder einen endlichen Grenzwert

$$
L_{\infty}=\frac{a}{1-b}
$$

Die von KNIGHT geleugnete Existenz eines Maximalwertes für das Längenwachstum geht also rein mathematisch aus der Guiltigkeit der FORD-WALFORD-Beziehung hervor. Diese ist nun dadurch interessant, daß sich aus ihr die BERTALANFFy-Funktion ableiten läßt. Aus dieser Beziehung leitete vor kurzem HoHENDORF (1966) ein einfaches Verfahren zur Berechnung von Näherungswerten für die Parameter der BERTALANFFYFunktion ab. Aus diesem Grunde kann man in ihr den Maximalwert der ForD-WaLFORD-Formel gebrauchen. Eine gewisse Differenz zwischen den beiden Funktionen liegt darin, daß die Regressionsberechnung für die ForD-WALFORD-Formel die Summe der Quadrate der linearen Abweichungen zum Mininum macht; dem Wesen des Wachstumsprozesses kommt man aber näher, wenn man die relativen Abweichungen zum Minimum macht.

Für die Daten von Ketchen \& Forrester (1966) für die 우우 von Eopsetta berechnete ich aus der FORD-WALFORD-Formel für die BERTALANFFY-Parameter $\mathrm{L}_{\infty}=$ 59,97 und für $K=0,162$. Es ergibt sich mit diesen Parametern - vor allem bei den ältesten Fischen - ein etwas besserer Anschluß an die Meßdaten, als ihn die Autoren erreichten. Die Differenz gegenüber dem von den Autoren vorgeschlagenen Maximalwert von $58,6 \mathrm{~cm}$ ist unbedeutend, vor allem da auch der Wert für $\mathrm{K}$ geändert wurde.

Eine andere Frage ist es, ob dem mathematischen Grenzwert eine biologische Bedeutung zukommt. Aus dem engen Zusammenhang zwischen Ford-WaLFord-Formel und BertalanfFY-Funktion (Krüger 1967) ergibt sich zwangsläufig, daß für beide Funktionen nahezu der gleiche Maximalwert gültig ist. Die hier vorliegende Ubereinstimmung kann man also nicht als Beweis für seine biologische Gültigkeit deuten.

Die Bertalanffy-Funktion liefert in der Mehrzahl der Fälle erstaunlich gute Annäherungen an gegebene Meßreihen, wie auch KNIGHT zugeben muß. Sie hat daker in der Fischereibiologie vielfache Anwendung erfahren. Sie stellt aber nicht die einzige Wachstumsfunktion dar, die vorgeschlagen wurde. KNIGHT erwähnt die logistische Funktion. Neben zahlreichen anderen wäre vor allem die GOMPERTz-Funktion zu nennen. Ich selbst habe vor einigen Jahren ebenfalls eine neue Funktion in Vorschlag gebracht (KRüGER 1962):

$$
y_{x}=\frac{y_{\max }}{N^{\frac{1}{x+\xi}}}
$$

Auch sie enthält einen Maximalwert. Grundsätzlich benötigt jede Wachstumsfunktion für ihre zahlenmäßige Auswertung eine Bezugsgröße, die entweder am Anfang der Wachstumsreihe steht, wie bei der Ford-WALFord-Formel oder es dient als Bezug die Maximalgröße, die sich bei der Extrapolation auf den Alterswert $\infty$ ergibt, wobei der zahlenmäßige Wert der Maxmialgröße von der mathematischen Interpretation der Wachstumskurve abhängt. Jede Funktion erfordert einen anderen Maximalwert. 
VON BertalanfFy (1934) war von dem Gedanken ausgegangen, in eine Wachstumsformulierung nur real beobachtete Zahlenwerte einzusetzen, erkannte aber sehr bald, daß etwas über den beobachteten Maximalwerten liegende Werte einen besseren Anschluß an gegebene Zahlenreihen gestatten. Damit führte er die Maximalgröße als rein mathematischen Parameter in seine Funktion ein. Für den praktischen Einsatz der Funktion erwies sich dieser Schritt als sehr wichtig, doch bestehen gegen die biologische Ausdeutung dieses Maximalwertes Bedenken. Auch in der von KNIGHT zitierten Untersuchung an Eopsetta jordani von KeTCHEN \& Forrester (1966) finden sich in den Tabellen für beide Geschlechter Individuen, die den aus den Durchschnittswerten errechneten Maximalwert beträchtlich überschreiten. Auch andere Literaturbeispiele zeigen, daß der Maximalwert der BerTalanfFY-Funktion keineswegs die obere Grenzgröße einer Art darstellt. Der Maximalwert der BERTALANFFY-Funktion hängt ab von den der Auswertung zugrundegelegten Durchschnittswerten, ist also von der Zusammensetzung der Zahlenreihen abhängig. Da aber der Maximalwert der BERTALANFFY-Funktion sich nicht sehr weit von real beobachteten Fischgrößen entfernt, erscheint seine Verwendung in weitergehenden Berechnungen durchaus vertretbar.

Der zahlenmäßige Wert der Maximalgröße hängt nun nicht nur von der Formulierung und der Zusammensetzung der Meßreihen ab, sondern schließlich auch noch von dem Verfahren, das man der Berechnung der Parameter zugrunde legt. Beruht dieses Verfahren auf einer linearen Annäherung an die Meßwerte, erhält man einen anderen Maximalwert, als wenn man eine relative Annäherung versucht. Immer stellt die Maximalgröße nur einen mathematischen Parameter dar. Auf die Diskussion der übrigen Parameter, die die spezielle Kurvengestalt bestimmen, möchte ich nicht eingehen. Gerade bei der BERTALANFFY-Funktion ist ihre Bedeutung wenig durchsichtig.

Aber auch wenn die Maximalgröße nicht einen ganz eindeutig zu bestimmenden Parameter darstellt, erfordert die zutreffende Beschreibung einer Wachstumsreihe die genaue Angabe der ermittelten Parameter - einschließlich des Maximalwertes. Aus diesem Grunde ist es nicht gleichgïltig, ob ich anstelle von $132 \mathrm{~cm} 130$ oder $135 \mathrm{~cm}$ angebe, auch wenn die wirkliche Schwankungsbreite wahrscheinlich noch größer ist.

Die primäre Aufgabe einer Wachstumsfunktion besteht darin, die Zahlenfolgen einer Wachstumsreihe möglichst gut zu simulieren und durch ihre Parameter womöglich den Kurvenverlauf zu charakterisieren. Eine weitere Aufgabe wäre es dann z. B., mit Hilfe der gewonnenen Parameter quantitative Einblicke in Okosysteme zu erhalten, wie es Beverton \& Holt (1957) versucht haben. Schon die erfolgreiche Lösung des ersten Problems stellt praktisch und wissenschaftich einen Gewinn dar, und die BERTALANFFY-Funktion löst diese Aufgabe für das Längenwachstum der Fische in recht guter Weise.

Die Bedenken gegen die BERTALANFFY-Funktion betreffen andere Eigenschaften. Sie enthält - ebenso wie die ForD-WALForD-Funktion - keinen Wendepunkt. Da das Längenwachstum der Fische im allgemeinen keinen Wendepunkt einschließt, wird ihre Anwendung dadurch kaum beeinträchtigt. Mit anderen Parametern läßt sich die BerTALANFFY-Funktion auch zur Beschreibung des Gewichtswachstums benutzen und gewinnt dann auch einen Wendepunkt, jedoch lassen sich die Gewichtsparameter nicht von den Längenparametern ableiten, sondern sind erneut zu berechnen. Das Maximalgewicht in der Gewichtsfunktion ist ein anderes, als es sich aus der maximalen Länge 
ergeben würde. Die Ursache liegt darin, daß die Parameter der Bertalanffy-Funktion keine definierte Beziehung zur allometrischen Funktion erkennen lassen. Daneben lassen auch andere Eigenschaften, auf die ich an dieser Stelle nicht eingehen möchte, die BERTALANFFy-Funktion in ihrer mathematischen Gestalt nicht als ideale Lösung erscheinen.

Der Vorzug der von mir vorgeschlagenen Wachstumsfunktion (Gleichung 5) liegt darin, daß sie in mathematisch definierter Beziehung zur allometrischen Funktion steht, so daß deren Parameter in sie eingesetzt werden können. Im Aufbau ähnelt der neue Vorschlag der BERTALANFFY-Funktion, während aber in dieser die im Verlauf des Wachstums eintretende Verminderung der Wachstumsraten durch den Abstand der gegebenen Dimension von der Maximalgröße beschrieben wird, übernimmt in der neuen Funktion ein reziproker Alterswert diese Aufgabe. Es muß hierbei allerdings der in den Tabellen angegebene Alterswert $(\chi)$ um einen konstanten Wert $(\xi)$ erhöht werden. Mathematisch ist der $\xi$-Wert ein Krümmungsparameter. Er schwankt für die verschiedenen Fischgattungen nur in relativ engen Grenzen und ist wenig kritisch für die numerischen Berechnungen. Bei der Unsicherheit aller Altersangaben ist diese Eigenschaft sehr vorteilhaft. In der zahlenmäßigen Auswertung ergibt sich eine mindestens ebensogute Annäherung an gegebene Wachstumsdaten wie bei der BERTALANFFY-Funktion. Die Parameter sind allerdings grundsätzlich abweichend, der Maximalwert liegt durchweg wesentlich höher.

\section{DIE MATHEMATISCHE WIEDERGABE DES WACHSTUMS VON EOPSETTA JORDANI}

Funktionen von mathematisch sehr unterschiedlicher Gestalt können in dem engen Bereich, der für Wachstumsauswertungen im allgemeinen zur Verfügung steht, einen sehr ähnlichen Verlauf zeigen. Für die Beurteilung der Güte der Wiedergabe von Wachstumsdaten eignen sich daher besonders Meßreihen, die sich über eine möglichst lange Altersspanne erstrecken. Aus diesem Grunde interessierten mich die Meßreihen von Ketchen \& Forrester an Eopsetta jordani, die bei den $q 9$ bei Berücksichtigung der kommerziellen Fänge 22 Altersstufen umfassen und bei den $\delta \hat{\delta}$ immerhin noch 17 Altersstufen.

Für die $Q O$ erreichten die beiden Autoren mit der BERTALANFFY-Funktion einen ausgezeichneten Anschluß an ihre Messungen. Ihren theoretischen Werten sind in Tabelle 1 die Zahlen gegenübergestellt, die sich nach der neuen Funktion ergeben, wenn man einen $\xi$-Wert von 1,7 zugrunde legt. Der zugehörige Maximalwert errechnet sich zu 73,9 $\mathrm{cm}$ und schließt damit auch jene Fische ein, die den Maximalwert der BERTALANFFY-Funktion $(60,0 \mathrm{~cm})$ überschreiten. Der größere Abstand des Maximalwertes von den Meßwerten hat auch noch den Vorteil, daß er durch Änderungen der beiden anderen Parameter nicht so stark beeinflußt wird.

Zur Beurteilung der Güte der Wiedergabe sind bei beiden Berechnungsweisen die prozentualen Abweichungen von den theoretischen Werten angegeben. In einzelnen Bereichen ergibt die BerTalanfFy-Funktion - in anderen Bereichen die neue Funktion eine bessere Näherung. Im ganzen gesehen, läßt sich trotz des weiten Meßbereiches, 
den dieses Beispiel bietet, kein erheblicher Unterschied im Kurvenverlauf und der Güte der Wiedergabe erkennen.

Ein weniger befriedigendes Ergebnis lieferte die BERTALANFFy-Funktion bei der Auswertung der Wachstumsdaten für die $\hat{\delta} \hat{\sigma}$, so daß die Autoren schon das Vorliegen

Tabelle 1

Vergleich der Wiedergabe der Meßwerte von KETCHen \& Forrester (1966) für die 9 ㅇ von Eopsetta jordani nach der BERTALANFFY-Funktion und der neuen Wachstumsfunktion

\begin{tabular}{|c|c|c|c|c|c|}
\hline \multirow[b]{2}{*}{ Alter } & \multirow[b]{2}{*}{$\begin{array}{l}\text { Meßwerte } \\
\text { Länge } \\
\text { (mm) }\end{array}$} & \multicolumn{2}{|c|}{ BERTALANFFY-Funktion } & \multicolumn{2}{|c|}{ Neue Wachstumsfunktion } \\
\hline & & $\begin{array}{c}\mathrm{L}_{\mathrm{o}}=600 \mathrm{~mm} \\
\mathrm{~K}=0,162 \\
\mathrm{t}_{0}=-0,2483\end{array}$ & $\begin{array}{l}\text { Abweichung } \\
\text { (in } \%)\end{array}$ & $\begin{aligned} \mathrm{L}_{\infty} & =73,89 \\
\log N & =2,21496 \\
\xi & =1,7\end{aligned}$ & $\begin{array}{l}\text { Abweichung } \\
\text { (in } \% / 0)\end{array}$ \\
\hline 1 & 110 & 110 & \pm 0 & 112 & $+1,8$ \\
\hline 2 & 190 & 183 & $-3,7$ & 186 & $-2,1$ \\
\hline 3 & 250 & 245 & $-2,0$ & 250 & \pm 0 \\
\hline 4 & 300 & 298 & $-0,7$ & 302 & $+0,7$ \\
\hline 5 & 345 & 344 & $-0,3$ & 345 & \pm 0 \\
\hline 6 & 385 & 382 & $-0,8$ & 381 & $-1,0$ \\
\hline 7 & 415 & 415 & \pm 0 & 411 & $-1,0$ \\
\hline 8 & 440 & 442 & $+0,4$ & 437 & $-0,7$ \\
\hline 9 & 460 & 466 & $+1,3$ & 459 & $-0,2$ \\
\hline 10 & 477 & 486 & $+1,9$ & 478 & $+0,2$ \\
\hline 11 & 492 & 503 & $+2,2$ & 495 & $+0,6$ \\
\hline 12 & 506 & 517 & $+2,0$ & 509 & $+0,6$ \\
\hline 13 & 519 & 530 & $+1,9$ & 522 & $+0,2$ \\
\hline 14 & 522 & 540 & $+3,5$ & 534 & $+2,3$ \\
\hline 15 & 545 & 549 & $+0,7$ & 544 & $-0,2$ \\
\hline 16 & 554 & 557 & $+0,5$ & 554 & \pm 0 \\
\hline 17 & 563 & 563 & \pm 0 & 563 & \pm 0 \\
\hline 18 & 572 & 569 & $=0,5$ & 570 & $=0,4$ \\
\hline 19 & 582 & 574 & $-1,4$ & 578 & $-0,7$ \\
\hline 20 & 573 & 577 & $+0,7$ & 584 & $+1,9$ \\
\hline 21 & 589 & 581 & $-1,4$ & 590 & $+0,2$ \\
\hline 22 & 599 & 584 & $-0,8$ & 596 & $-0,5$ \\
\hline
\end{tabular}

von Meßfehlern in Betracht ziehen. Bei der FORD-WALFORD-Darstellung fallen die Punkte für die drei ersten Altersstufen aus der geradlinigen Anordnung der übrigen Meßpunkte heraus. Daher war zu erwarten, daß sie sich auch durch die BertalanffyFunktion nicht befriedigend darstellen lassen, was die Zahlen der Autoren in Tabelle 2 bestätigen.

Im Gegensatz hierzu ließen sich die Meßwerte für die $\hat{\partial} \hat{o}$ beinahe ebensogut wiedergeben wie für die 우, wie die geringen Abweichungen in Tabelle 2 zeigen. In diesem Falle weichen in den Altersstufen 1 bis 3 die beiden Funktionen stark voneinander $\mathrm{ab}$. Zwischen den Altersstufen 4 und 17 verlaufen sie wieder parallel. Wenn im allgemeinen auch die Ergebnisse der beiden Funktionen nahe beieinanderliegen, erweist sich in diesem Falle die neue Wachstumsformulierung als anpassungsfähiger.

Ich kann nur versuchen, die an dieser Stelle auftretende Frage zu beantworten, weshalb in diesem Falle ein so ausgeprägter Unterschied im Verlauf der beiden Funktionen auftritt. Als Grundlage für die Parameterrechnung erhielt ich als Näherungswert 0,7 für $\xi$ für das Wachstum der $\delta \delta$. Bei den $q q$ betrug der $\xi$-Wert 1,7. Wenn 
auch im allgemeinen bei den Fischen der $\xi$-Wert für die $q Q$ höher ist als bei den $\hat{\partial} \hat{\partial}$, so habe ich einen so starken Geschlechtsunterschied bislang noch nicht getroffen.

Der niedrige Wert für den Krümmungsparameter $\xi$ besagt, daß wir es bei den ô ô mit einem Wachstum zu tun haben, das anfänglich sehr stark ist, dann aber schon

\section{Tabelle 2}

Vergleich der Wiedergabe der Meßwerte von KETCHEN \& Forrester (1966) für die $\hat{\delta} \delta$ von Eopsetta jordani nach der BERT'ALANFFY-Funktion und der neuen Wachstumsfunktion

\begin{tabular}{|c|c|c|c|c|c|}
\hline \multirow[b]{2}{*}{ Alter } & \multirow{2}{*}{$\begin{array}{l}\text { Meßwerte } \\
\text { Länge } \\
(\mathrm{mm})\end{array}$} & \multicolumn{2}{|c|}{ BERTALANFYY-Funktion } & \multicolumn{2}{|c|}{ Neue Wachstumsfunktion } \\
\hline & & $\begin{array}{c}\mathrm{L}_{\infty}=490 \mathrm{~mm} \\
\mathrm{~K}=0,160 \\
\mathrm{t}_{0}=-2,60\end{array}$ & $\begin{array}{l}\text { Abweichung } \\
\text { (in } \% / 0)\end{array}$ & $\begin{array}{c}\mathrm{L}_{\infty}=55,72 \\
\log N=1,23822 \\
\xi=0,7\end{array}$ & $\begin{array}{l}\text { Abweichung } \\
\text { (in } \% \text { ) }\end{array}$ \\
\hline 1 & 105 & 215 & & 104 & $-1,0$ \\
\hline 2 & 185 & 250 & & 194 & $+4,9$ \\
\hline 3 & 260 & 285 & & 258 & $-0,8$ \\
\hline 4 & 315 & 315 & \pm 0 & 304 & $-3,6$ \\
\hline 5 & 346 & 345 & $-0,3$ & 338 & $-2,3$ \\
\hline 6 & 368 & 366 & $-0,5$ & 364 & $-1,1$ \\
\hline 7 & 385 & 385 & \pm 0 & 385 & \pm 0 \\
\hline 8 & 401 & 400 & $=0,2$ & 402 & $+0,2$ \\
\hline 9 & 414 & 413 & $-0,2$ & 415 & $+0,2$ \\
\hline 10 & 425 & 425 & \pm 0 & 427 & $+0,5$ \\
\hline 11 & 434 & 434 & \pm 0 & 437 & $+0,7$ \\
\hline 12 & 442 & 443 & $\mp 0,2$ & 445 & $+0,7$ \\
\hline 13 & 450 & 450 & \pm 0 & 453 & $+0,7$ \\
\hline 14 & 458 & 456 & $=0,4$ & 459 & $+0,2$ \\
\hline 15 & 463 & 461 & $-0,4$ & 465 & $+0,4$ \\
\hline 16 & 468 & 465 & $-0,6$ & 470 & $+0,4$ \\
\hline 17 & 473 & 469 & $-0,8$ & 474 & $+0,2$ \\
\hline
\end{tabular}

auf frühen Altersstufen stark abklingt. Die harmonische Reihe, die der Kurvenbeschreibung der neuen Funktion zugrunde liegt, gestattet in ihrem Anfangsteil die Darstellung eines solch starken Abfalls. Die BerTALANFFY-Funktion scheint diesen steilen Abfall nur in beschränktem Umfang wiedergeben zu können. Der $\xi$-Wert der 우오 liegt mit 1,7 in der Größenordnung, die bei den meisten Fischen angetroffen wird, auf die die BERTALANFFY-Funktion mit Erfolg angewandt werden konnte.

Eine Eigentümlichkeit, die Wachstumskurven mit niederem $\xi$-Wert auszeichnet, ist die Erfahrung, daß sich bei ihnen der Maximal-Wert sehr stark den höchsten Meßwerten nähert. Dadurch rückt er in die Nähe des Maximalwertes der BertalanffyFunktion. Im Fall der $\delta \hat{\delta}$ von Eopsetta beträgt der Maximalwert $55,7 \mathrm{~cm}$ gegenïber $49,0 \mathrm{~cm}$ bei der BERTALANFFY-Funktion. In diesem Falle nähern sich also mathematische Berechnungen und biologische Beobachtung weitgehend. Auch die Wachstumsdaten der $\delta \hat{o}$ von Eopsetta lassen also auf der Basis der neuen Wachstumsfunktion durchaus eine mathematische Interpretation zu. Ich glaube daher nicht, daß den Autoren bei ihrer offensichtlich außerordentlich sorgfältigen Arbeit Fehler unterlaufen sein könnten. Die Kontrolle von Meßreihen anhand mathematischer Wachstumsmodelle ist - wie man sieht - ein weiterer Gesichtspunkt, der für die Ausarbeitung möglichst leistungsfähiger Formulierungen spricht. 
Trotz der sehr erheblichen Kritik von KNIGHT (1968) liegen solche Lösungen schon vor. Man kann nur feststellen, daß dieser Autor sich viel zuwenig mit den Lösungen und Problemen der Wachstumsmathematik beschäftigt hat. Aus diesem Grunde war es auch sehr leicht, seine Kritik an der Annahme eines oberen Grenzwertes beim Fischwachstum zu widerlegen, ebenso wie andere Thesen, die seine Veröffentlichung enthält.

Es ist sehr zu begrüßen, wenn sich Mathematiker der vielen Probleme annehmen, welche die Anwendung mathematischer Verfahren in der Biologie liefert. Eine ganz oberflächliche Behandlung der Probleme und Ignorierung aller biologischer Gegebenheiten dient aber niemandem; sie kann nur Verwirrung stiften, nicht aber als eine fruchtbare Diskussionsbasis dienen.

\section{ZUSAMMENFASSUNG}

1. KNIGHT (1968) äußerte aufgrund einer oberflächlichen Auswertung einer unvollständigen Zahlenreihe für das Wachstum des Kabeljau (Gadus morbua) die Ansicht, daß für das Fischwachstum kein oberer Grenzwert existiert und daher die Einsetzung eines solchen in eine mathematische Wachstumsformulierung ein Nonsens sei.

2. Nun ist aber die Annäherung jeden Fischwachstums an eine artgemäße Endgröße wie bei allen Tieren - eine bekannte Tatsache. Der asymptotische Charakter dieser Annäherung geht mathematisch aus der FoRD-WALFORD-Beziehung hervor, deren Gültigkeit entgegen der Ansicht von KNIGHT als gesichert angesehen werden muß.

3. Die in eine Wachstumsfunktion eingesetzte Maximalgröße stellt in jedem Falle einen mathematischen Parameter dar, der von der Formulierung, den Meßdaten und der Berechnung abhängt. Die biologische Deutung dieses Maximalwertes ist daher unsicher.

4. Eine von KNIGHT zitierte Untersuchung von KeTCHEN \& ForRester (1966) uber das Wachstum von Eopsetta jordani wird zur Prüfung der neuen Wachstumsfunktion benutzt. Sie liefert - besonders deutlich erkennbar bei den $\hat{\partial} \hat{\partial}$ - eine wesentlich bessere Annäherung an die gegebenen Daten als die BertalanfFy-Funktion.

\section{ZITIERTE LITERATUR}

Bertalanffy, L. von, 1934. Untersuchungen über die Gesetzlichkeit des Wachstums. Allgemeine Grundlagen der Theorie. Arch. EntwMech. Org. 131, 613-653.

- 1938. A quantitative theory of organic growth. Hum. Biol. 10, 181-213.

Beverton, R. J. H. \& Holt, S. J., 1957. On the dynamics of exploited fish populations. Fishery Invest., Lond. (Ser. 2) 19, 1-533.

FORD, E., 1933. An account of the herrings investigations conducted at Plymouth during the years from 1924-1933. J. mar. biol. Ass. U. K. 19, 305-384.

Graham, M., 1933. Report on the North sea cod. Fishery Invest., Lond. (Ser. 2) 13, 1-160.

Hansen, P. M., 1949. Studies on the biology of the cod in Greenland waters. Rapp. P.-v. Réun. Cons. perm. int. Explor. Mer 123, 1-77.

HOHENDORF, K., 1966. Eine Diskussion der BERTALANFFY-Funktion und ihre Anwendung zur Charakterisierung des Wachstums von Fischen. Kieler Meeresforsch. 22, 70-97. 
Ketchen, K. S. \& Forrester, C. R., 1966. Population dynamics of the petrale sole, Eopsetta jordani, in waters off western Canada. Bull. Fish. Res. Bd Can. 153, 1-195.

KNIGHT, W., 1968. Asymptotic growth: an example of nonsense disguised as mathematics. J. Fish. Res. Bd Can. 25, 1303-1307.

KRÜGER, F., 1962. Uber die mathematische Darstellung des tierischen Wachstums. Naturwissenschaften $49,454$.

- 1965. Zur Mathematik des tierischen Wachstums. I. Helgoländer wiss. Meeresunters. 12, $78-136$.

- 1967. BertalAnFFY-Funktion und FORD-WALFORD-Formel. Helgoländer wiss, Meeresunters. 16, 193-206.

WALFORD, L. A., 1946. A new method of describing the growth of animals. Biol. Bull. mar. biol. Lab., Woods Hole 90, 141-147.

Anschrift des Autors: Prof. Dr. F. KrüGER

Biologische Anstalt Helgoland

Zentrale

2 Hamburg 50

Palmaille 9 